\title{
Off-Set Filtering for Enhanced Transmission in RSOA Based WDM-PON
}

\author{
A. Gatto ${ }^{1}$, P. Parolari ${ }^{*}$, L. Marazzi ${ }^{1}$, M. Brunero ${ }^{1}$, S. Barbet ${ }^{2}$, A. Maho ${ }^{2}$, R. Brenot ${ }^{2}$, G. Gavioli ${ }^{3}$, P. Galli $^{3}$, \\ and M. Martinelli ${ }^{1}$ \\ ${ }^{1}$ Politecnico di Milano, Dip. Elettronica Informazione e Bioingegneria, Milano, 20133 Italy \\ ${ }^{2}$ III-V Lab, joint lab of 'Alcatel-Lucent Bell Labs France', 'Thales Research and Technology' and 'CEA Leti', \\ Marcoussis, 91460 France \\ ${ }^{3}$ Alcatel-Lucent Italia, Vimercate, Milano, Italy \\ *Tel: +3902 23998941, e-mail: parolari@elet.polimi.it
}

\begin{abstract}
We present an experimental analysis on externally seeded colourless transmitters for WDM PON. We take advantage of direct modulation of a high performance MQW RSOA. Chirp is analysed as main limiting factor to SSMF propagation at $10 \mathrm{~Gb} / \mathrm{s}$. We demonstrate that off-set filtering performed by the OLT demultiplexer allows to cope with chromatic dispersion impairments and to bridge $24-\mathrm{km} \mathrm{SSMF}$ at $10 \mathrm{~Gb} / \mathrm{s}$ with modest penalties with respect to back to back.
\end{abstract}

Keywords: WDM PON, seeded RSOAs, filter detuning, chirp, performance analysis, colourless transmitter.

\section{INTRODUCTION}

Next generation access networks (NGAN) will probably gain great advantages in exploiting the potentialities of wavelength division multiplexing (WDM) in passive optical networks (PON) [1]. It is widely acknowledged that the success of this proposal will essentially depend on the capability to obtain scale economies mainly by developing the same optical network unit (ONU) for all the users and by thoroughly deploying the fibre optical bandwidth. This means that ONU's should be colour-agnostic: colourless ONU's can be obtained by the injection technique performed on Fabry-Perot lasers [2] and reflective semiconductor optical amplifiers (RSOA) [3] or even in Self-seeded RSOA [4]. RSOAs are preferred devices for many solutions exploiting a seeding source placed at the optical line terminal (OLT), which propagates in the same downstream (DS) direction, but in another bandwidth with respect to upstream (US), e.g. DS and upstream US wavelengths respectively in L and C bands. In particular due to usual band limitation of standard RSOA, to target high bit rates such as $10 \mathrm{~Gb} / \mathrm{s}$, various techniques have been demonstrated. Electronic equalization and Forward Error Correction (FEC) $[5,6]$, off-set optical filtering aided by electronic equalization [7], detuned ad-hoc delay interferometers [8] and recently off-set filtering directly realized by a common WDM demultiplexer [9] proved the capacity to successfully target tens of kilometres feeder link with ONU directly modulated RSOAs.

In this paper we present a new high performance RSOA, which shows improved E/O bandwidth, nevertheless due to chirp associated with direct modulation, the propagation at high bit rates is limited by the interaction with chromatic dispersion. Off-set filtering is successfully exploited to increase the system tolerance towards dispersion penalties, targeting up to 24-km standard single-mode fiber (SSMF) feeder link at $10 \mathrm{~Gb} / \mathrm{s}$.

\section{EXPERIMENTAL RESULTS}

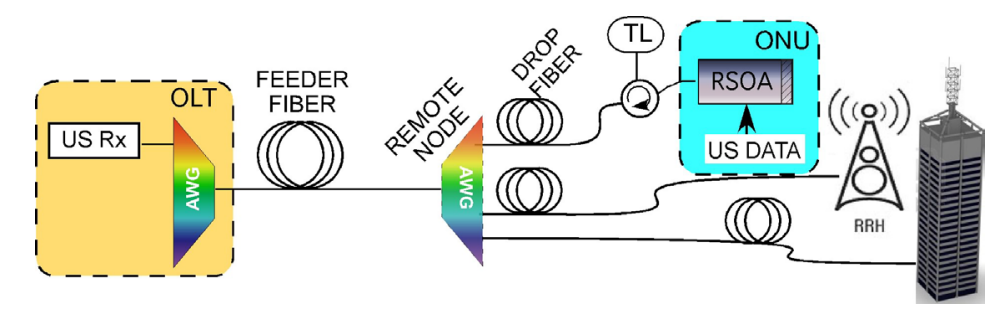

Figure 1. Experimental set up.

The experimental set up, which implements an externally seeded WDM-PON transmitter, is described in Fig. 1. The CW external seeding is generated by a Tunable Laser (TL) to allow for transmitter signal detuning with respect to AWG transfer function. The RSOA seeding power is kept at $-6 \mathrm{dBm}$, and is fed by an optical circulator. Polarization controllers maximize the signal input to the RSOA, which is a HPDG compressively strained multiple quantum well (MQW) RSOA. The RSOA has a measured E/O bandwidth of approximately $4 \mathrm{GHz}$, at $100 \mathrm{~mA}$ bias current, it presents the gain peak at $1530 \mathrm{~nm}$ and the measured small signal gain is $30 \mathrm{~dB}$. The RSOA amplifies and reflects the $\mathrm{CW}$ seeded signal and the upstream signal is obtained by directly modulating its bias current. 


\subsection{Chirp analysis}

Direct current modulation is associated with a carrier density variation, which causes a chirp accompanying the transmitted signal. As the RSOA is primarily an amplifier the chirp contribution is transient and concordant with the intensity modulation. At first chirp measurements have been performed to measure the frequency variation contribution. In order to obtain it the frequency discriminator method has been exploited employing two linear filters, with opposite angular coefficients and sufficient bandwidth to discriminate the expected chirp [10]. Figure 2(a) shows the typical signal measured optical spectrum (red line) and the filter transfer functions with bandwidth of $50 \mathrm{GHz}$ (blue line), exploited to measure the chirp associated with the RSOA high-bit rate operation. After the filtering, the two signals are detected by a linear photodiode and they are acquired by a digital sampling oscilloscope (DSO); the real-time chirp measurements are obtained by post-processing the sampled data.

In Fig. 2(b) frequency chirp (blue line) and intensity (red line) measurements relative to a specific intensity pattern are shown for the RSOA injected by a CW seed generated by the TL. The RSOA is directly modulated at $10 \mathrm{~Gb} / \mathrm{s}$. The device is biased at $100 \mathrm{~mA}$ and the amplitude modulation is $2 \mathrm{~V}_{\mathrm{pp}}$, obtaining an US extinction ratio of $5 \mathrm{~dB}$. The limited bandwidth of the RSOA is highlighted in the intensity reduced dynamic of ' 101 ' and ' 010 ' transitions. Figure 2(b) confirms that the adiabatic chirp which refers to a shift between the emission frequency of the two signal levels, e.g. mark and spaces, is almost zero, whereas a transient chirp appears at the rising and falling edges of the modulated signal and is related to the first derivative of the output power. A "positive chirp" is observed for increasing output power and a negative frequency variation is observed for decreasing output power. During transmission over SSMF the US signal is subject to positive dispersion thus the positive chirp speeds up the pulse broadening impairing the transmission performance.

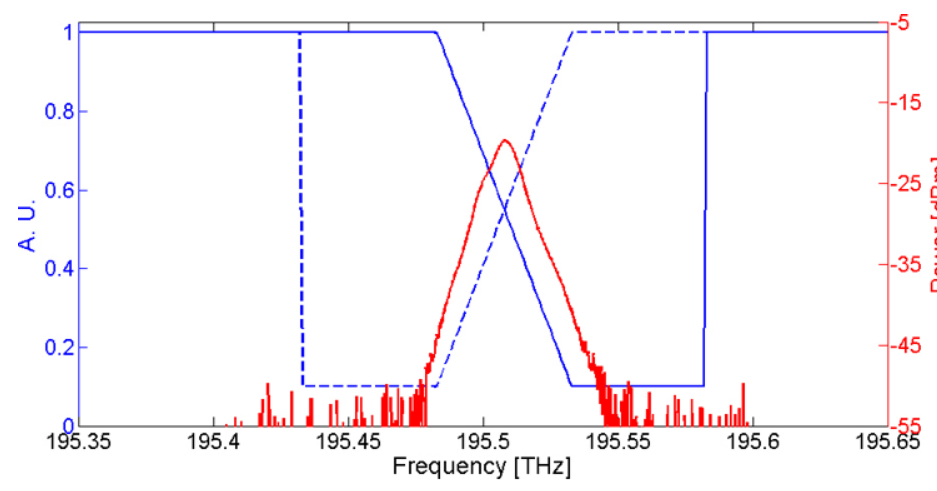

(a)

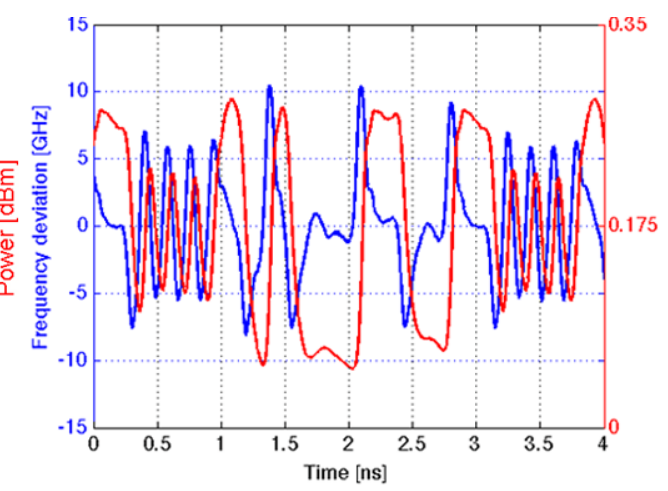

(b)

Figure 2:(a) signal measured optical spectrum (red line) and transfer function of the linear filters (blue line) for chirp measurements; (b) measured frequency chirp (blue) and intensity (red).

In the above conditions at $10 \mathrm{~Gb} / \mathrm{s}$ the strong transient component shows a peak-to-peak chirp of about 18 GHz. As the transient chirp depends on the first time derivative of the emitted power, its time duration and magnitude are determined by the electro-optical bandwidth of the device. Lower bandwidths lead to a limited value of the magnitude while the time duration (to which is proportional the inter symbol interference (ISI)) increases; higher bandwidths, instead, lead to a higher magnitude but in a short time period. This chirp drastically limits the propagation reach of the system to a few kilometers, but a proper off-set filtering could improve the transmission performance. Moreover, Fig.2(b) shows that positive and negative frequency deviations at the rising and falling edges, respectively, have similar magnitude, that is in principle both positive and negative off-sets can be exploited. In the next section, the detuning between the $\mathrm{CW}$ seeding frequency and the AWG centre frequency is expressed as: $\Delta f=f_{A W G}-f_{\text {seed }}$. For positive frequency detunings, thus the signal lower frequencies are reduced, while for negative detunings the signal higher frequency components are limited.

\subsection{0-Gb/s transmission}

The externally seeded WDM-PON transmitter has been tested at $10 \mathrm{~Gb} / \mathrm{s}$ with increasing dispersive loads, i.e. with different lengths of SSMF. In order to evaluate the improvement of the propagation performance for different detunings of the optical filter with respect to the signal frequency, a programmable filter that mimics a 55-GHz Gaussian athermal AWG transfer function has been exploited as OLT demultiplexer. The US receiver is an avalanche photodiode (APD) with a $10-\mathrm{Gb} / \mathrm{s}$ sensitivity of $-26 \mathrm{dBm}$ for 13-dB extinction ratio signals. The APD is followed by a clock and data recovery (CDR) with equalization capabilities, exploiting a 9-taps feed forward equalization (FFE) and a 4-taps decision feedback equalization (DFE) filters.

At first we present back to back BER measurements for various filter detunings (Fig. 3a). Contrary to [9] RSOA O/E bandwidth allows for error free performance also with no off-set filtering, it can be observed in fact 
that no significant improvement is present in back to back also for high detunings, as bandwidth limitation is modest and equalization unnecessary.

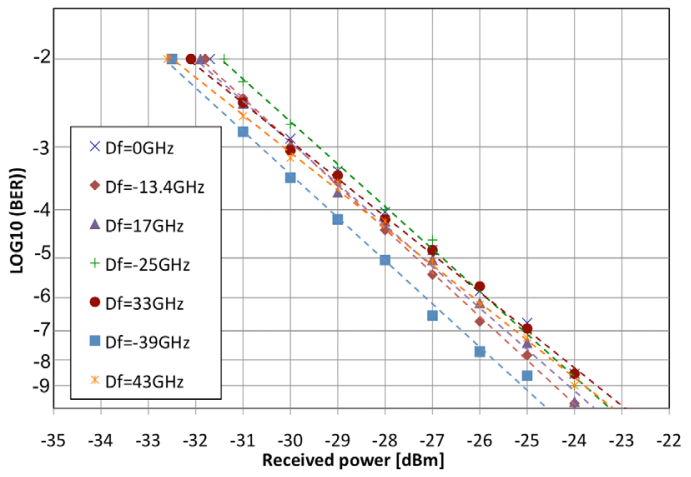

(a)
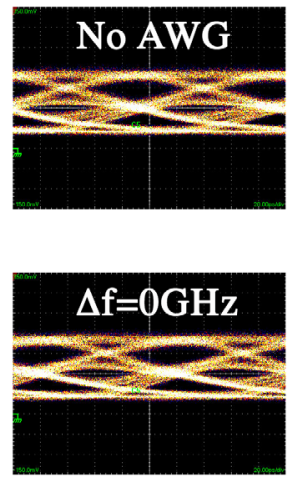
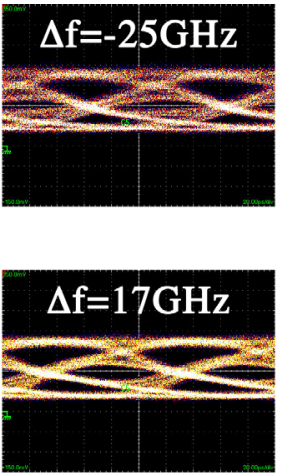

(b)

Figure 3: a) BER for back to back configuration for positive and negative detunings;

b) eye diagrams with increasing off-set after 10-km SSMF propagation.

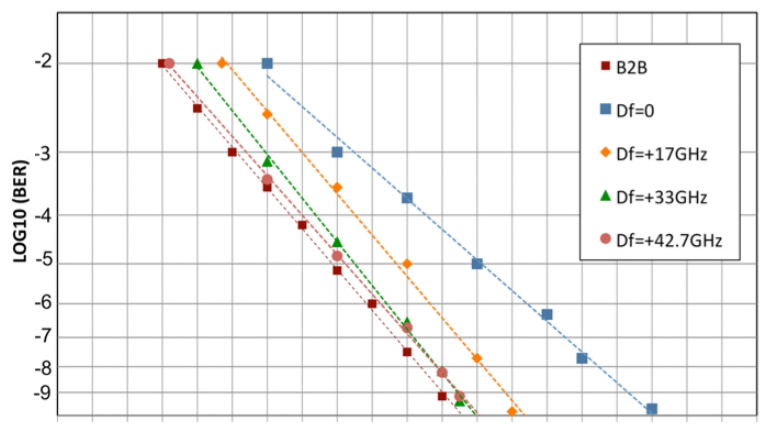

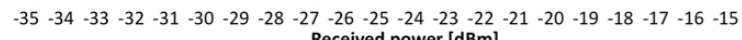

(a)

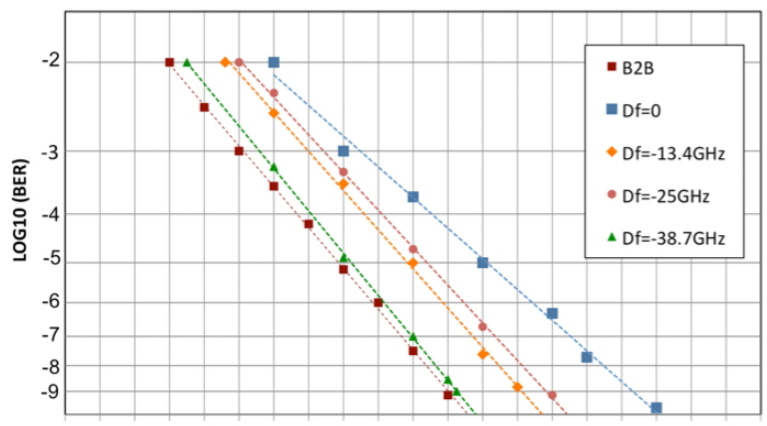

$\begin{array}{lllllllllllllllllllll}-35 & -34 & -33 & -32 & -31 & -30 & -29 & -28 & -27 & -26 & -25 & -24 & -23 & -22 & -21 & -20 & -19 & -18 & -17 & -16 & -15\end{array}$

(b)

Figure 4. Propagation after 10-km SSMF at $10 \mathrm{~Gb} / \mathrm{s}$ : a) BER for positive frequency detuning,

b) BER for negative frequency detuning.

In Fig. $3 b$ the eye diagrams and BER measurements for different frequency detuning after 10-km SSMF propagation are shown. If no filter is inserted, the received signal is deteriorated by ISI due to chromatic dispersion. As expected, no change is visible after the introduction of a centred filter ( $0 \mathrm{GHz}$ detuning) because of the large bandwidth of the AWG filter. Signal quality improvement can be clearly seen when increasing the frequency detuning between the seed and the AWG central frequency. In particular, the eye is almost open for frequency detuning of $-38 \mathrm{GHz}$ and $+43 \mathrm{GHz}$, although the eye shape isn't the same, depending on the opposite PM to AM conversion realized by the two slopes of the AWG filter. In Fig.4(a) and 4(b) the BER performance for positive and negative frequency detuning are reported. A significant improvement of the system performance with respect to the null detuning can be seen for large frequency detunings: the chromatic dispersion penalty with respect to the back to back curve is reduced from $4 \mathrm{~dB}$ to almost zero. For detunings higher than $\Delta f= \pm 30 \mathrm{GHz}$ no significant improvement is visible, while the filter attenuation increases from $3 \mathrm{~dB}$ to $8.7 \mathrm{~dB}$ at $\Delta f= \pm 40 \mathrm{GHz}$. A good trade-off between power penalties due to filtering effect and system performance increase due to filter off-set is represented by the $15-\mathrm{GHz}$ frequency detuning, which leads to a $1.5 \mathrm{~dB}$ penalty with $0.5 \mathrm{~dB}$ power losses.

Figure 5(a) shows eye diagrams and BER performance for different frequency detuning for a 24-km SSMF propagation at $10 \mathrm{~Gb} / \mathrm{s}$. Differently from the $10-\mathrm{km}$ propagation, an open eye diagram can be seen only for very large frequency detunings, i.e. for $\Delta f=-38 \mathrm{GHz}$ and $\Delta f=+43 \mathrm{GHz}$. The high transient chirp component, in fact, leads to a complete deterioration of the transmitted signal after $24 \mathrm{~km}$ of SSMF. Without the demultiplexing filter no BER can be evaluated, as for a perfectly centred filter. Increasing the frequency detuning, the BER curves get closer to the back to back curve. As reported in Fig. 5(b), a minimum penalty of about $2 \mathrm{~dB}$ can be achieved for a detuning of $\Delta f=+43 \mathrm{GHz}$, which introduces an unavoidable power loss of $8.7 \mathrm{~dB}$ due to $\mathrm{AWG}$ attenuation. A good trade off can be considered a frequency detuning of about $30 \mathrm{GHz}$, which leads to a $5 \mathrm{~dB}$ penalty with almost $3 \mathrm{~dB}$ extra power losses. 

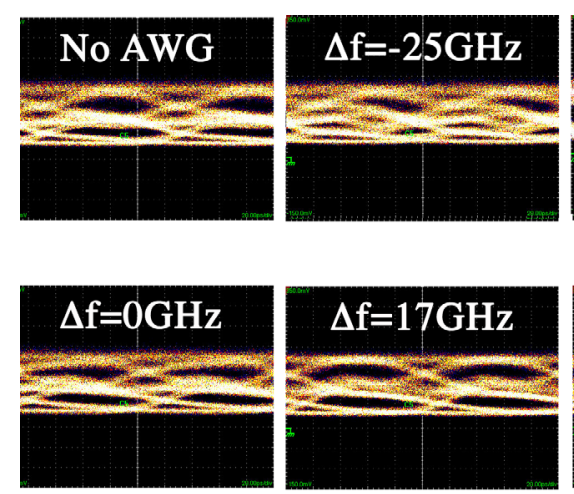

(a)
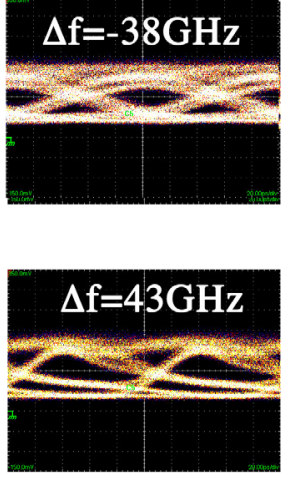

Figure 5. Propagation

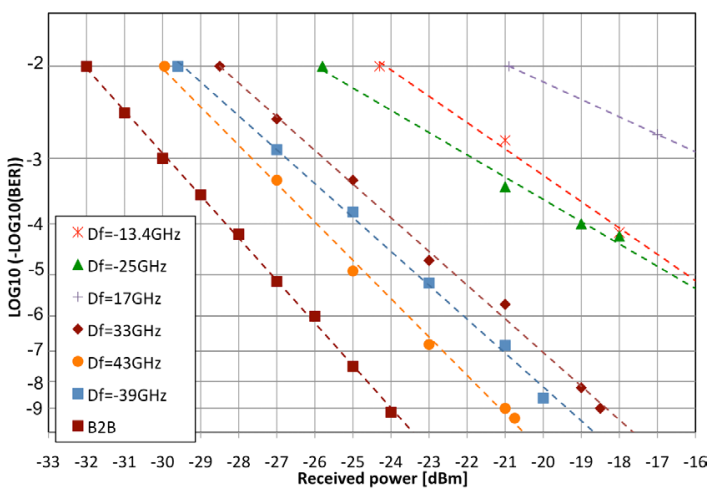

(b)

b) BER for positive and negative frequency detuning.

For the $24 \mathrm{~km}$ propagation it's clearly visible that only very high detunings permit the transmission with admissible power penalties at the receiver. Contrary to [9] where off-set filtering is already necessary even in back to back due to RSOA bandwidth limitations, here filter detunings reduce CD impairments allowing for tens of kilometres propagation, moreover as PM to AM conversion is not essential both positive and negative detunings can be adopted.

\section{CONCLUSIONS}

We presented an experimental analysis on chirp components associated with externally seeded RSOAs, exploited for ONU colourless transmitters for WDM PONs. Contrary to previously demonstrated band-limited RSOA back to back equalization is not necessary due to the high performance MQW RSOA. Nevertheless chirp associated with direct modulation represents a limiting factor for SSMF propagation at $10 \mathrm{~Gb} / \mathrm{s}$. We demonstrated that thanks to OLT demultiplexer detuning we can cope with chromatic dispersion impairments obtaining error free transmission up to $24-\mathrm{km} \mathrm{SSMF}$ at $10 \mathrm{~Gb} / \mathrm{s}$. A proper trade-off between best detuning for $\mathrm{CD}$ overcoming and off-set power penalty allows for enhanced 10-Gb/s operation.

\section{ACKNOWLEDGEMENTS}

The research leading to these results has received funding from the European Union's Seventh Framework Programme (FP7/2007-2013) under grant agreement ERMES n 2885422012 and from the Italian Ministry of University and Research through ROAD-NGN project (PRIN2010-2011).

\section{REFERENCES}

[1] C.-H. Lee, Sang-Mook Lee, K.-M. Choi, J.-H. Moon, S.-G. Mun, K.-T. Jeong, J. H. Kim, B. Kim, "WDMPON experiences in Korea [Invited]", J. Opt. Netw. vol. 6, pp. 451-464, 2007.

[2] N. Kashima, "Dynamic properties of FP-LD transmitters using side mode injection locking for LAN's and WDM-PONs", J. Lightwave Technol., vol. 24, pp. 3045-3058, Aug. 2006.

[3] S. J. Park, Y-B Choi, J-M Oh, S-G Koo, D. Lee., "An evolution scenario of a broadband access network using R-SOA-based WDM-PON technologies", J. Lightwave Technol. vol. 25, pp. 3479-3487, Nov. 2007.

[4] L. Marazzi, P. Parolari, M. Brunero, A. Gatto, M. Martinelli, R. Brenot, S. Barbet, P. Galli, G. Gavioli "Up to 10.7-Gb/s high-PDG RSOA-based colorless transmitter for WDM networks, ,IEEE Photon. Technol. Lett, vol. 25, pp. 637-640, Apr. 2013.

[5] A. Agata, Y. Horiuchi, "R-SOA based 10G WDM-PON using FEC and MLSE equalizers", in Proc. OFCNFOEC 2010, paper OWG3.

[6] B. Schrenk. G de Valicourt, M. Omella, J. A. Lazaro, R. Brenot, J. Prat, "Direct 10-Gb/s modulation of a single-section RSOA in PONs with high optical budget”, IEEE Photon. Technol. Lett., 22, Mar. 2010.

[7] H. Kim, "10-Gb/s operation of RSOA using a delay interferometer", IEEE Photon. Technol. Lett., 22, Mar. 2010.

[8] H. Kim, "Transmission of 10-Gb/s directly modulated RSOA signals in single-fiber loopback WDM PONs", IEEE Photonics Technology Letters, vol. 22, no. 14, Jul. 15, 2011.

[9] M. Presi, A. Chiuchiarelli, R. Corsini, P. Choudury, E. Ciaramella "Enhanced 10-Gb/s operation of bandwidth-limited R-SOAs without electronic equalization", in Proc. ECOC 2012, Amsterdam, paper We.1.B.2, 2012.

[10] R. T. Watts, K. Shi, L. P. Barry, "Time-resolved chirp measurement for 100GBaud test systems using an ideal frequency discriminator”, Opt. Comm., 285, pp. 2039-2043, Apr. 2012. 